\title{
Análisis y medida de la ubicuidad y usabilidad de los portales web de las universidades españolas
}

\author{
Francisco Javier Martínez Méndez
}

Universidad de Murcia (España)

\section{Resumen}

Los portales web de las universidades españolas son pioneros en el desarrollo de sistemas de información en nuestro país. Tras más de una década desde su inicio, este conjunto de sedes sigue poseyendo interés y presencia destacados, y ha suscitado el desarrollo de diversos estudios que han medido algunos aspectos parciales y puntuales de su desarrollo e implementación. Este estudio engloba aspectos vinculados a la ubicuidad, visibilidad, organización de los contenidos, aspectos formales y presencia de servicios de fidelización con el objetivo de ponderar debidamente la importancia de cada uno de ellos en una fórmula final. Nuestro propósito es definir medidas que garanticen la viabilidad de futuras repeticiones de este análisis, evitando así su obsolescencia. El resultado refleja globalmente el grado de desarrollo general y permite la construcción de un portal que servirá de observatorio para el análisis y estudio detallado de esta cuestión.

Palabras clave: Universidades. World Wide Web. Portales. Ubicuidad. Usabilidad web. Visibilidad. Accesibilidad web. Metadatos. Organización de contenidos. Motores de Búsqueda. España.

\section{Abstract}

The web sites of the Spanish universities can be considered pioneers in the development of information systems in our country. A decade after their beginning, these sites still have an outstanding presence and interest, having motivated the development of several studies that have measured specific aspects of their implantation. This analysis includes a wide range of aspects related to the ubiquity, visibility, content organization, formal aspects and users' faithfulness, with the aim to properly reflect the importance of each one of them in a final grid. Our purpose is to define a set of measures that guarantee the viability of future replications of this experiment. The results reflect the degree of development of these sites and have been the background of a web portal that will serve as an observatory for the analysis and detailed study of this question.

Scire. 14 : 2 (jul.-dic. 2008) 87-106. ISSN 1135-3716. 
Keywords: Universities. World Wide Web. Web sites. Ubiquity. Visibility. Web usability. Web accessibility. Metadata. Contents organization. Search engines. Spain.

\section{Introducción}

Los portales de las universidades españolas son los sitios web con más antigüedad de España. Cuando se puso en marcha este servicio en Internet como medio de difusión del conocimiento, las universidades españolas lo acogieron fervorosamente y contribuyeron enormemente a su popularización. Desde el inicio, estos sitios cumplieron dos funciones: difusión de la información institucional y creación, publicación, diseminación y uso compartido del conocimiento en todos los ámbitos científicos. Por ello, no es atrevido afirmar que estos portales son pioneros en el desarrollo de sistemas de información en la Web. Estos sitios siguen manteniendo una presencia considerable dentro de la Web española por varias razones: están dirigidos a un público muy habituado al uso de las tecnologías de la información, alojan una ingente cantidad de páginas, muchas de ellas de incontestable valor técnico o científico, y en estas instituciones se hace un uso generalizado de la Web para mantener puntualmente informados a sus miembros y como apoyo de las actividades docentes y de investigación. Según datos de la CRUE, "más del $60 \%$ de las Universidades Españolas prestan servicios directos a través del portal a la comunidad universitaria. Por lo tanto, hoy día el reto para las universidades no es estar en la red, sino cómo estar presentes en ella" (Duart, 2006).

En líneas generales, el grado de desarrollo de estos portales es medio o elevado. Sus diseños varían en función de las instituciones aunque pueden identificarse algunos elementos comunes: $a$ ) la mayoría ofrecen productos y servicios, en muchos casos dentro de entornos dinámicos de programación y consulta a bases de datos; b) suelen establecer perfiles/secciones en función de grupos de usuarios (alumnosprofesores-personal-visitantes); c) se habilita un acceso a intranet restringido a los miembros de la comunidad universitaria; $d$ ) en la mayoría de los casos se ha desarrollado una imagen corporativa, y e) los servicios de acceso a la información se han convertido en convertido en elementos fijos e indiscutibles en la mayoría de estos portales.

No todos sus diseñadores han prestado la misma atención a algunas de las cuestiones que más interés suscitan hoy en día: la buscabilidad del portal, la visibilidad del sitio para facilitar la adquisición de la información, la organización de su contenido y la disposición de elementos que faciliten el acceso a los mismos, entroncando con la usabilidad y la accesibilidad, sin olvidar la disposición de servicios de valor añadido que generen en el usuario la necesidad de volver a hacer uso del portal. Una combinación adecuada de todos estos aspectos contribuye a la conformación de un producto final suficientemente válido que satisfará las necesida-

Scire. 14 : 2 (jul.-dic. 2008) 87-106. ISSN 1135-3716. 
des de sus usuarios. Desafortunadamente, la realidad muestra día a día pocos ejemplos de esa debida (y deseable) amalgama.

Por su importancia consideramos pertinente llevar a cabo un trabajo de análisis y revisión de todos estos conceptos, con el fin de percibir el actual estado de la cuestión. En su desarrollo se intentará obviar —en la mayor medida posible— la presencia de elementos subjetivos vinculados al diseño de un portal, procurando considerar únicamente aspectos objetivos de medida directa, con el fin de proponer una fórmula final que permita construir una clasificación (ranking) de los distintos portales. Este trabajo nace con una vocación de continuidad, lo que obliga a considerar un conjunto de parámetros cuya revisión sea viable, algo totalmente necesario en el contexto de la Web.

\section{Objeto de interés del estudio}

En los últimos años asistimos a la eclosión del interés por la usabilidad de las páginas web propiciada por el tremendo impacto de los trabajos de Rosenfeld y Morville (2000) y de Nielsen y Tahir (2002). Sus aportaciones son la base los estudios de usabilidad y de organización de los contenidos. Se trata de "un atributo de calidad que mide lo fáciles de usar que son las interfaces web" (Nielsen, 2003). Su revisión se realiza por medio de una exhaustiva heurística de gran coste que no todas las organizaciones están dispuestas a asumir. Muchos administradores de sitios Web no suelen plantearse si sus contenidos resultan fácilmente localizables ni si, una vez localizados, resultan accesibles (un documento no accesible o difícilmente accesible pierde su esencia y función), de modo que los contenidos adquieran un nuevo atributo, el de la efectividad.

Como bien recuerda García Gómez (2006), "es más barato construir una web usable y accesible desde el principio que hacerlo después”. Esto justifica la profusión de trabajos recomendando criterios básicos para el diseño de webs usables, con la idea de no tener que invertir posteriormente en procesos de rediseño, mucho más costosos. En esta línea apreciamos el trabajo de Baeza-Yates y Rivera-Loaiza (2002), quienes exponen cinco ideas claves para el diseño efectivo de sitios web: $a$ ) buscabilidad, $b$ ) visibilidad, $c$ ) contenido, $d$ ) estética y $e$ ) fidelidad. Los autores intentan dar respuesta a una pregunta básica: “¿qué es más importante, la forma o el contenido?". Lo normal sería decir que el contenido; sin embargo, "en realidad no importa si no podemos llegar a él. Por eso es que mis mandamientos para la Web tienen un orden causal y cada uno de ellos puede tener la misma importancia, pero sin el primero no tenemos el segundo, sin el segundo el tercero, etcétera. Hago énfasis en los dos primeros, que son los menos conocidos, pero todos ellos están basados en el sentido común".

¿Qué elementos de las páginas web intervienen directamente en la buscabilidad de las páginas? Los anteriormente citados Baeza-Yates y Rivera-Loaiza aportan

Scire. 14 : 2 (jul.-dic. 2008) 87-106. ISSN 1135-3716. 
varios: que la dirección del sitio web esté debidamente registrada en los motores, que el diseño no ponga trabas a los robots, que en la página principal aparezca el título del sitio web (sorprende encontrar sitios web donde no aparece un texto indicando su nombre o bien se usa incorrectamente). La ubicuidad se refleja en su posicionamiento en buscadores (no confundir con popularidad o impacto). En la mejora de la ubicuidad de un sitio web convergemos necesariamente con los metadatos, aunque su uso en la web es bastante desigual y está ausente en algunos casos. Esta ausencia se debe sin duda alguna a la desidia de los diseñadores, actitud que no viene siendo corregida del todo por los propios motores de búsqueda, que aún parecen no valorarlos en su justa medida.

El segundo factor que participa en la ubicuidad es la visibilidad. Tiene que ver con la posibilidad de acceder correctamente al contenido una vez localizado. Entre sus indicadores destacan el tamaño óptimo de la página, un diseño compatible y líquido, un aprovechamiento de la interfaz según los estándares más aceptados y el factor de impacto web, introducido por Almind e Ingwersen y basado en el análisis de enlaces (LabInternet, 2006). Si bien esta medida se encuentra sujeta a algunas críticas, parece correcta, aunque debería darse más peso a la visibilidad y segregar contenidos según su impacto potencial. Existe un grupo de páginas cualitativamente más importantes ("ficheros ricos" en formato pdf, doc, ppt o ps) que están más relacionadas con la actividad de publicación y merecen, por tanto, un tratamiento diferenciado. Esta idea permite introducir en una medida meramente cuantitativa algunos conceptos cualitativos.

Baeza-Yates y Rivera-Loaiza (2002) miden la usabilidad prestando especial atención a tres factores: organización del contenido, aspectos estéticos y fidelización del usuario. Evidentemente los autores simplifican mucho esta tarea (normalmente realizada a partir de la observación detallada de una cantidad muy superior de factores, muchos de ellos subjetivos). Valorar la organización del contenido a través de una serie de factores clave y objetivos no es sencillo (acudimos a la presencia de elementos que ayuden al visitante en la navegación o en la recuperación de información dentro del portal).

Otro concepto evaluable - y que cada día cobra mayor trascendencia- es la accesibilidad. Garantizar un diseño accesible es algo que necesariamente deben satisfacer los diseñadores de sitios web, con el fin de realizar una plena efectividad y universalidad en la gestión de los contenidos. En realidad, la mayoría de los problemas de accesibilidad son fácilmente subsanables por parte de los diseñadores, que pueden orientar sus hábitos hacia un diseño accesible sin excesivo esfuerzo.

Si bien algunos autores se sienten capaces de valorar cuantitativamente aspectos estéticos del diseño de una página, consideramos esta categoría como susceptible de verse afectada por la subjetividad del evaluador. Por ello se prefiere obviar su revisión. Por último queda valorar el grado de fidelización del portal, que

Scire. 14 : 2 (jul.-dic. 2008) 87-106. ISSN 1135-3716. 
puede ponderarse por su tráfico y por la presencia de servicios de valor añadido que generen "algo que se necesite en forma periódica, que sea adictivo" (Baeza-Yates y Rivera-Loaiza, 2002).

\section{Estado de la cuestión}

Se dispone de estudios previos que cubren algunas de las áreas comentadas y que sirven de referencia para el desarrollo de nuestra investigación. El primero es el realizado por Gil Leiva y Moya Martínez (2001), que analiza 17 sitios web (uno por comunidad autónoma) revisando indicadores de contacto, legibilidad y ergonomía, navegación y representación, recuperabilidad, velocidad de descarga y luminosidad. Los resultados principales obtenidos dibujaban un panorama poco alentador.

Más exhaustivo y elaborado es el trabajo llevado a cabo por un grupo de trabajo dirigido por la profesora Pinto Molina sobre la visibilidad de la investigación de 19 universidades españolas a través de sus páginas web (Pinto Molina et álii, 2004). En este trabajo "se decidió considerar la visibilidad en tres niveles. En primer lugar, en cuanto a presencia, esto es, en la medida en que la información que es consistentemente objeto de interés por parte de los usuarios está efectivamente disponible. En segundo lugar, en cuanto a calidad, a saber, el grado en el que dicha información es completa, multimedia, veraz y actual, a la vez que está convenientemente autorizada. Y, en tercer lugar, en cuanto a usabilidad, esto es, hasta qué punto dicha información se presenta adecuadamente, para que sea usable por los usuarios". Resulta muy coincidente con nuestros planteamientos la frase "la detección de indicadores cualitativos ha abierto la puerta al análisis de indicadores cuantitativos — susceptibles de ser recogidos por un robot de búsquedas- que correlacionen bien con medidas de calidad" (Pinto Molina et álii, 2004). Los autores consideran totalmente necesaria la presencia de un mapa del sitio e importante el hecho de que los sitios web estén suficientemente reconocidos por las instituciones por medio de un texto de autoría/responsabilidad en la página o por medio de logotipos, así como un seguimiento exhaustivo de las pautas de accesibilidad web dictadas por el World Wide Web Consortium (2005).

Hacia finales del año 2005 se publicó el Proyecto Usabilidad Páginas Web Universidades Españolas (USABAIPO, http://griho.udl.es/aipo/que_es_aipo.jsp [2005-11-20]), que analizaba las páginas de inicio de 69 universidades españolas. Este trabajo inspecciona el cumplimiento de estándares en los portales de las universidades, el análisis de las zonas de la interfaz del usuario y el desarrollo de evaluaciones heurísticas. La mayoría de los indicadores utilizados se diseñaron por las recomendaciones de Nielsen y Tahir (2002). La fecha de actualización del estudio y su amplia cobertura (casi todas las universidades) sirven de fuente de referencia directa y actualizada. Destaca especialmente el análisis de la zona de interfaz

Scire. 14 : 2 (jul.-dic. 2008) 87-106. ISSN 1135-3716. 
realizado, porque el aprovechamiento del espacio de una página y la distribución de los elementos principales se convierte en un factor de calidad y, lo que es más importante, puede medirse cuantitativamente. Los autores establecen una medida de la distribución de la interfaz a partir de las diferencias entre la distribución de cada portal con la ideal.

En lo concerniente con la accesibilidad de las páginas web de las universidades españolas, se dispone del informe realizado en agosto de 2004 por el observatorio disc@apnet. Es una evaluación técnica de la accesibilidad de 15 universidades que ha sido posteriormente actualizado con una segunda versión (febrero de 2006). Lo mejor de este informe es "la recogida de datos doble, un test heurístico y una encuesta de experiencia de usuarios, no se limita solo a pasar una revisión automática” (García Gómez, 2005). Lo peor, evidentemente, es su escasa cobertura (no llega a la cuarta parte del total de universidades) y el descorazonador panorama que ofrecen los resultados: ninguno de esos centros supera la evaluación.

Englobado en un proyecto de extensión geográfica mundial, recientemente se ha presentado el denominado "Ranking cibermétrico de las web de Universidades Españolas", publicado en la web webometrics.info, y desarrollado por el grupo de trabajo del CINDOC InternetLab. Este trabajo combina una serie de indicadores complementarios que ponderan diversos aspectos de su presencia en la web y se ordenan de acuerdo con un indicador que "combina el volumen de información publicada, la visibilidad e impacto de estas páginas según el número de enlaces externos que reciben" (LabInternet, 2006). Esta medida, aunque sujeta a algunas críticas, parece correcta, pero, siguiendo en cierto modo la línea del trabajo anterior (Pinto Molina et álii, 2004), LabInternet se adentra un poco en los aspectos cualitativos (en el contenido de los documentos), apostando por dar más peso a la visibilidad y segregar contenidos según su impacto potencial.

Confirmando el creciente interés de los investigadores de nuestra área de conocimiento (y más concretamente de los investigadores españoles), la Universitat Oberta de Catalunya ha editado un monográfico dedicado a la presencia de las universidades en Internet. En el mismo encontramos tres trabajos interesantes: uno sobre los antecedentes y las características principales de las revistas científicas digitales (Abadal y Rius, 2006), otro dedicado a los conceptos y modelos de los portales en la educación superior (Franklin, 2006) y un tercer trabajo elaborado por Aguilló y Granadino Goenechea sobre los indicadores web empleados en la construcción del ranking cibermétrico anteriormente comentado.

Desafortunadamente, no se dispone de datos concretos sobre el uso de los metadatos en las páginas de inicio de las universidades españolas (en este punto particular, nuestro estudio podría considerarse pionero). 


\section{Objetivos}

Nuestro trabajo de investigación pretende alcanzar los siguientes objetivos: $a$ ) identificar los aspectos de ubicuidad y usabilidad de los portales web que puedan convertirse en indicadores cuantitativos de la calidad y el grado de desarrollo de los mismos; $b$ ) partiendo de esos indicadores, revisar el estado actual de la cuestión en la totalidad de los portales de las universidades españolas; $c$ ) fijar una fórmula que integre los resultados parciales de cada categoría de indicadores en un ranking final, ponderando debidamente los pesos de cada uno de ellos, y d) habilitar un espacio en la web que sirva a la vez como observatorio de los resultados del estudio y como repositorio de documentos.

De la medida de la ubicuidad de estos portales se obtendrán una serie de objetivos específicos hasta ahora inalcanzados: $e$ ) conocer el nivel de la presencia de los metadatos en la definición de las páginas principales de las universidades españolas; $f$ ) revisar la accesibilidad de la totalidad de las páginas principales de todas las universidades, y $g$ ) analizar si existen algunas tendencias claramente diferenciadas en el diseño y gestión de los portales en función de la naturaleza de la institución académica.

\section{Metodología}

Para llevar a cabo este trabajo de investigación se han desarrollado una serie de etapas en diversos períodos de tiempo, aplicando un riguroso procedimiento normalizado de observación y medida: 1) recopilación de las direcciones de las páginas principales; 2) cuando esas direcciones consistían en menús de acceso sin contenidos, se procedió a cambiar esa dirección por la de primera página con contenidos del portal; 3) en el caso de aquellas universidades con versiones en varios idiomas (algo cada vez más habitual por la proyección internacional de estas instituciones y por el bilingüismo presente en gran parte de España) se optó por analizar la primera de las opciones; 4) establecimiento del conjunto de indicadores a analizar y del procedimiento para tomar la medida en cada caso particular; 5) toma de datos, y (6) se realizó una segunda revisión de la accesibilidad de los portales para detectar el grado de mejora.

\subsection{Fechas de realización del estudio}

El trabajo de investigación se ha desarrollado en tres períodos de tiempo: $a$ ) el grueso de la recogida de datos se llevó a cabo a lo largo del mes de noviembre de $2005 ; b$ ) en la última semana de enero de 2006 se tomaron los datos de buscadores y sindicación de contenidos, y c) durante los primeros días de febrero de 2006 se actualizaron datos concernientes a la accesibilidad de estos portales (aunque apenas se detectaron sustanciales cambios, coincidiendo con la revisión de dic@pnet de febrero 2006).

Scire. 14 : 2 (jul.-dic. 2008) 87-106. ISSN 1135-3716. 


\subsection{Procedimiento de observación y medida}

Se analizaron por separado los cuatro grandes apartados de nuestro estudio: buscabilidad y visibilidad (que conforman la ubicuidad), y organización de contenidos y fidelización (que conforman la usabilidad).

Medida de la buscabilidad. Se analizan cinco parámetros en esta categoría: a) buscador encuentra mi sitio (1 punto); $b$ ) ausencia de obstáculos para el robot (1 punto); $c$ ) texto de la organización claramente ubicado (1 punto); d) posición del portal en buscadores ( 2 puntos), y $e$ ) metadatos de contenidos y de propiedad intelectual (2 puntos).

\begin{tabular}{|c|c|c|}
\hline Parámetro & Peso & Procedimiento de medida \\
\hline $\begin{array}{l}\text { a) Buscador encuentra } \\
\text { mi sitio }\end{array}$ & 1 & $\begin{array}{l}\text { Se busca por el nombre de la universidad el portal en Google, } \\
\text { Yahoo! y MSN. Si aparece en la primera posición de la respuesta } \\
\text { esta medida sería 1, si estuviera en la segunda posición valdría } \\
0,66, \text { y en la tercera, } 0,33 \text {. En otra posición o en ausencia vale } 0 \text {. } \\
\text { Se calcula el valor medio de la puntuación individualmente } \\
\text { obtenida en cada motor. }\end{array}$ \\
\hline $\begin{array}{l}\text { b) Ausencia de obstáculos } \\
\text { para el robot }\end{array}$ & 1 & $\begin{array}{l}\text { Se revisa la página de inicio. Si aparece uno de estos obstáculos } \\
\text { (flash o mapas de bits) se asigna un } 0,5 \text {. Si su frecuencia de } \\
\text { aparición es superior a } 1 \text { se asigna un valor igual a } 0 \text {. En caso } \\
\text { de no aparecer se asigna un } 1 \text {. }\end{array}$ \\
\hline $\begin{array}{l}\text { c) Texto de la organización } \\
\text { claramente ubicado }\end{array}$ & 1 & $\begin{array}{l}\text { Se localiza en la página principal el nombre completo y } \\
\text { correctamente escrito de la universidad (si se trata de un gráfico } \\
\text { se verifica el texto alternativo). Si está presente se asigna un } 1 \text {, } \\
\text { aunque si no se identifica claramente la institución por medio de } \\
<\text { TITLE se le asigna únicamente } 0,5 \text {. En caso de no aparecer el } \\
\text { texto en la página se asigna un valor de } 0 \text {. }\end{array}$ \\
\hline $\begin{array}{l}\text { d) Posición del portal } \\
\text { en buscadores }\end{array}$ & 2 & $\begin{array}{l}\text { Se busca "universidad" en los tres motores anteriores y se anota } \\
\text { el ordinal de la posición en la respuesta. Al primero se le asigna } \\
\text { un valor de } 2 \text { puntos y progresivamente se reduce el peso hasta } \\
\text { llegar a } 0 \text { puntos en el último lugar (o no si no llega a aparecer } \\
\text { en las diez primeras páginas de la respuesta). Se calcula el valor } \\
\text { medio obtenido en cada motor. }\end{array}$ \\
\hline $\begin{array}{l}\text { e) Metadatos de } \\
\text { contenidos y de } \\
\text { propiedad intelectual }\end{array}$ & 2 & $\begin{array}{l}\text { Se revisa la presencia de metadatos en la página de inicio } \\
\text { identificando los de contenido, instanciación y propiedad. Los } \\
\text { portales con presencia aceptable de metadatos de contenido o } \\
\text { propiedad intelectual tendrán } 1 \text { punto asignado. }\end{array}$ \\
\hline
\end{tabular}

Tabla I. Medida de la buscabilidad.

A partir de los valores individuales asignados a cada parámetro, la buscabilidad se calculará aplicando la siguiente fórmula:

$$
\text { buscabilidad }=(a) *[(a)+(b)+(c)+(d)+(e)]
$$

La suma total de los valores de los parámetros individuales se multiplica por el primero de ellos con el fin de penalizar a aquellos portales que no aparezcan en

Scire. 14 : 2 (jul.-dic. 2008) 87-106. ISSN 1135-3716. 
los tres primeros lugares de la respuesta. Se considera un error grave que incide directamente en la buscabilidad de un sitio web. El valor máximo posible de esta medida es 7 puntos.

Medida de la visibilidad. Se analizan cuatro parámetros: $f$ ) tamaño óptimo de la página de inicio (2 puntos); $g$ ) diseño compatible y líquido (1 punto); $h$ ) análisis de las zonas de la interfaz de usuario ( 2 puntos), y, finalmente, $i$ ) factor de impacto (2 puntos).

\begin{tabular}{|c|c|c|}
\hline Parámetro & Peso & Procedimiento de medida \\
\hline $\begin{array}{l}\text { f) Tamaño óptimo de la } \\
\text { página de inicio }\end{array}$ & 2 & $\begin{array}{l}\text { Se calcula el peso real de descarga de la página de inicio. Si el } \\
\text { tamaño es menor o igual a } 25 \mathrm{~kb} \text { el valor de la medida es } 2 \text {; si el } \\
\text { peso oscila entre } 25 \text { y } 50 \mathrm{~kb} \text { se asigna una valor de } 1 \text {. Un peso } \\
\text { superior obtendrá un valor igual a } 0 \text {. }\end{array}$ \\
\hline $\begin{array}{l}\text { g) Diseño compatible } \\
\text { y líquido }\end{array}$ & 1 & $\begin{array}{l}\text { Se verifica (1) el diseño compatible y (2) el diseño líquido. La } \\
\text { compatibilidad Windows-Macintosh se comprueba por medio del } \\
\text { revisor safaritest. En caso de no existir problemas el valor de la } \\
\text { medida es } 1 \text {. Si existieran diferencias pequeñas, la medida } \\
\text { valdría } 0,5, \text { y en caso de diseño no compatible el valor será } 0 \text {. El } \\
\text { diseño líquido de la página se verifica cambiando la resolución } \\
\text { de la pantalla. En caso afirmativo la medida valdrá } 1 \text {, si se } \\
\text { detectan pequeñas incorrecciones la medida valdrá } 0 \text {. En caso } \\
\text { muy negativo, valdrá } 0 \text {. }\end{array}$ \\
\hline $\begin{array}{l}\text { h) Análisis de las zonas } \\
\text { de la interfaz de usuario }\end{array}$ & 2 & $\begin{array}{l}\text { A partir de la distribución por zonas de la interfaz de la página } \\
\text { principal, el proyecto USABAIPO elabora una fórmula ponderada } \\
\text { del parecido con una distribución estándar. Se ha normalizado } \\
\text { esa función a un rango de } 0 \text { a } 2 \text { puntos. }\end{array}$ \\
\hline i) Factor de impacto & 2 & $\begin{array}{l}\text { Se consulta la posición de cada universidad en el ranking } \\
\text { webometrics.info. Al primero de los sitios web se le asigna un } \\
\text { valor igual a } 2 \text { puntos; a continuación decrece el peso de esta } \\
\text { medida hasta llegar a } 0 \text { puntos en el caso de la universidad que } \\
\text { aparezca en último lugar (o no llegue a aparecer en el ranking). }\end{array}$ \\
\hline
\end{tabular}

Tabla II. Medida de la visibilidad.

$$
\text { visibilidad }=(\mathrm{f})+(\mathrm{g})+(\mathrm{h})+(\mathrm{i})
$$

El valor máximo posible de esta medida es 7 puntos. 
Medida de la organización. La forman tres parámetros: $j$ ) mapa web y elementos de navegación ( 2 puntos); $k$ ) buscador web (2 puntos), y, finalmente, $l$ ) accesibilidad (2 puntos).

\begin{tabular}{|l|c|l|}
\hline Parámetro & Peso & Procedimiento de medida \\
\hline $\begin{array}{l}\text { j) Mapa web y elementos } \\
\text { de navegación }\end{array}$ & 2 & $\begin{array}{l}\text { Se verifica la existencia de mapa web o de otro elemento de } \\
\text { ayuda en la navegación. Si existen, el valor será de 2 puntos. Si } \\
\text { solo existe uno de estos elementos, el valor de la medida será 1, } \\
\text { y en caso de inexistencia, será 0. }\end{array}$ \\
\hline k) Buscador web & 2 & $\begin{array}{l}\text { Se verifica la existencia y la posición de buscador interno. Si se } \\
\text { ubica en la zona superior derecha se le asigna un valor de 2 } \\
\text { puntos. Si se encontrara en el resto de la zona superior o en la } \\
\text { centro-derecha valdría 1. Otras ubicaciones que dificultan su } \\
\text { visibilidad, o la inexistencia del mismo, implicarán un valor de 0. }\end{array}$ \\
\hline I) Accesibilidad & 2 & $\begin{array}{l}\text { Se revisa la accesibilidad del sitio web y se verifica el } \\
\text { cumplimiento de la especificación AA (satisfacción de los dos } \\
\text { primeros niveles de accesibilidad). En caso afirmativo se asigna } \\
\text { un valor de 2. Cumplir la especificación vale 1 punto. No } \\
\text { satisfacer ninguna especificación equivale a un valor nulo } \\
\text { de esta medida. }\end{array}$ \\
\hline
\end{tabular}

Tabla III. Medida de la organización.

A partir de los valores individuales asignados a cada parámetro, la visibilidad se calculará aplicando la siguiente fórmula:

$$
\text { organización }=(\mathrm{j})+(\mathrm{k})+(\mathrm{i})
$$

El valor máximo posible de esta medida es 6 puntos. Esta medida admitiría una revisión conducente a potenciar el papel del parámetro accesibilidad, revisión que incluiría el cumplimiento de las especificaciones AAA (satisfacción de todos los niveles de las pautas de accesibilidad web). 
Medida de la fidelización. Se han determinado cinco parámetros: $m$ ) densidad del tráfico (2 puntos); $n$ ) servicios de comunicación (1 punto); $\tilde{n}$ ) servicios de acceso a la información (1 punto); o) servicios de difusión de la información (1 punto), y $p$ ) servicios de personalización (1 punto).

\begin{tabular}{|l|c|l|}
\hline Parámetro & Peso & Procedimiento de medida \\
\hline m) Densidad del tráfico & 2 & $\begin{array}{l}\text { Se toman los datos de la variable traffic rank de alexa.com. Se } \\
\text { calcula el intervalo entre el mayor y menor y se normalizan los } \\
\text { datos originales para el intervalo de 0 a 2 puntos, evitando } \\
\text { favorecer en exceso a los portales de gran tamaño. }\end{array}$ \\
\hline $\begin{array}{l}\text { n) Servicios de } \\
\text { comunicación }\end{array}$ & 1 & $\begin{array}{l}\text { Se revisa la posibilidad de acceder desde la página principal al } \\
\text { servidor de correo electrónico de la universidad o a un campus } \\
\text { virtual o intranet. Si ambas opciones son posibles el valor de la } \\
\text { medida es 1; si solo es posible una de ellas la medida tendrá de } \\
\text { valor 0,5. En caso de no existir estos enlaces el valor es 0. }\end{array}$ \\
\hline $\begin{array}{l}\text { n) Servicios de acceso } \\
\text { a la información }\end{array}$ & 1 & $\begin{array}{l}\text { Se revisa la presencia en la página de inicio de apartados de } \\
\text { novedades, noticias, biblioteca, directorio, boletines de prensa, } \\
\text { radio o televisión Si todos son accesibles el valor de la medida } \\
\text { es 1. El mismo decrecerá proporcionalmente por cada ausencia. }\end{array}$ \\
\hline $\begin{array}{l}\text { o) Servicios de difusión } \\
\text { de la información }\end{array}$ & 1 & $\begin{array}{l}\text { Se verifica si desde la página principal se ofrece un servicio de } \\
\text { difusión selectiva de la información o de sindicación de } \\
\text { contenidos. El valor de esta medida será igual a 1. En caso } \\
\text { contrario será igual a 0. }\end{array}$ \\
\hline $\begin{array}{l}\text { p) Servicios de } \\
\text { personalización }\end{array}$ & 1 & $\begin{array}{l}\text { Se verifica si el sitio web establece perfiles de usuario (PAS, PDI, } \\
\text { alumnos, visitantes). En caso afirmativo se asigna medio punto. } \\
\text { Si además permitiera un servicio de personalización tipo "Mi } \\
\text { portal" se asignaría otro medio punto. En caso de inexistencia } \\
\text { de estos servicios el valor sería igual a 0. }\end{array}$ \\
\hline
\end{tabular}

Tabla IV. Medida de la fidelización.

A partir de los valores individuales asignados a cada parámetro, la fidelización se calculará aplicando la siguiente fórmula:

$$
\text { fidelización }=(m)+(n)+(\tilde{n})+(o)+(p)
$$

El valor máximo posible de esta medida es 6 puntos.

La medida final de la ubicuidad y usabilidad es la suma de las cuatro medidas anteriores.

$$
\mathrm{U}^{2}=\text { buscabilidad + visibilidad + organización + fidelización }
$$

En función de los valores máximos que cada una de las medidas posee, estas tienen una relevancia dentro de la fórmula final, que podemos ver representada en el gráfico de la figura 1. 


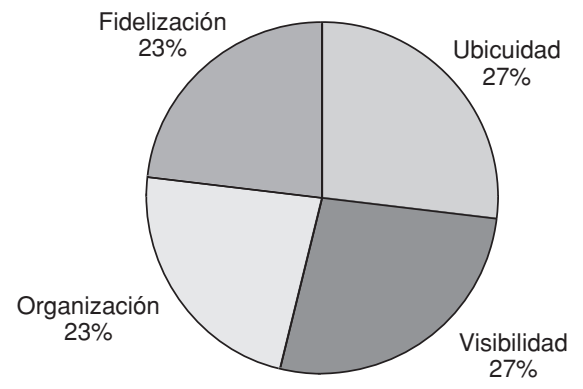

Figura 1. Porcentajes de la fórmula final $U^{2}$.

\section{Resultados concernientes a la buscabilidad}

Tanto en Google como en Yahoo! todas las universidades aparecen en primer lugar de la respuesta. No podemos decir lo mismo respecto al buscador MSN. El uso de mapas de bits en las páginas de inicio es una técnica erradicada. Esto no ocurre con la tecnología flash, que está presente en un tercio de los portales, y mucho más frecuentemente en los portales de las universidades privadas. El texto con el título de la organización está claramente ubicado en casi todas las páginas de inicio analizadas.

Sería lógico suponer que la totalidad de los portales de las universidades españolas aparecen entre las diez primeras páginas de la respuesta de cada buscador a la pregunta "universidad". La realidad nos ha demostrado una situación ligeramente diferente. Algunas universidades tienen serios problemas de visibilidad.

La frecuencia de aparición de metadatos obtenida ha resultado pobre y diversa. Su rango oscila desde un elemento (el título de la página web generalmente) hasta 26 (Universidad de León), y se ha verificado la existencia de un portal sin metadatos (Universidad Francisco de Vitoria). Es preciso indicar que el revisor considera como metadatos las clásicas metaetiquetas del lenguaje HTML, sin limitar este concepto a las del esquema Dublin Core (si así lo hubiéramos hecho, el número sería mucho menor). Las frecuencias de aparición de los metadatos de contenido varían mucho de un elemento a otro. La información sobre el título de la página aparece en casi todos los casos. Este profuso uso no es trasladable al resto de metadatos: las palabras clave (subject) y descripción (description) solo alcanzan una presencia relativa (alrededor del 50\% de los casos), y resulta increíble que el elemento idioma (language) solo figure en una cuarta parte de los portales analizados, máxime si tenemos en cuenta el uso de varias lenguas oficiales dentro de nuestro Estado. El resto de los metadatos de esta tipología no se usa. La presencia de los metadatos de propiedad en las páginas web de las universidades muestra unas frecuencias que, en el mejor de los casos, se podrían considerar relativamente es-

Scire. 14 : 2 (jul.-dic. 2008) 87-106. ISSN 1135-3716. 
casas (el de mayor frecuencia no llega al 30\%), de modo que la mayoría de los elementos de esta categoría resultan prácticamente testimoniales. Es especialmente difícil de entender esta situación en tanto que estas páginas representan a instituciones académicas y científicas, la mayoría de ellas oficiales - que deberían preocuparse por proteger la propiedad intelectual de la información recogida en ellas, o, al menos, refrendarla-, por medio de estos elementos de metadatos. No obstante, la realidad es harto diferente. La revisión de los metadatos de instanciación muestra unas distribuciones de frecuencias variadas. La fecha del recurso aparece en más o menos la mitad de los casos, aunque no aporta apenas información representativa. El tipo de recurso se encuentra definido en todos los casos (página HTML, dato normalmente introducido de forma automática por los editores de la página web). El resto de los elementos apenas son considerados.

Se observa un uso escaso de la metaetiqueta "robot", situación deseable porque no insertar nada equivale a la directiva "ALL". Esta metaetiqueta está bien empleada en los portales de las universidades españolas.

\section{Resultados concernientes a la visibilidad}

En cuanto al tamaño de la página de inicio, la realidad no es muy halagüeña. Solo una pequeña proporción (alrededor del 13\%) de las páginas estudiadas poseen un diseño óptimo o aceptable. En lo relativo al diseño líquido y al diseño compatible, el análisis ha revelado que no todos los portales se encuentran diseñados para una plena configuración del entorno operativo desde el que se accede. Esto acarreará sin duda algunos problemas de accesibilidad a los sitios web y, por lo tanto, dificultará la recuperación de la información en ellos contenida. En el análisis de la zona de interfaz, y como cabía esperar, el nivel de desajuste entre las proporciones ideales de aprovechamiento de esta zona y los diseños finales del usuario es bastante elevado.

En lo relativo a la posición en el ranking de webometrics.info se observa que las universidades públicas muestran unos valores ligeramente más altos que las privadas, con la excepción de la Universidad de Navarra. Esto parece lógico por el tamaño de las organizaciones y por el mayor número de "ficheros ricos" presentes en el sector público.

\section{Resultados concernientes al contenido}

Se emplea más el mapa web (especialmente en el caso de las universidades privadas) que la barra de navegación. Este elemento está más presente en las universidades públicas que en las privadas. Otro factor que se ha de destacar es la posición en la página (está asumido como estándar que la mejor posición es la zona superior de la ventana alineada a la derecha, por ser el primer lugar hacia donde dirigimos nuestra mirada). Esto se cumple en el $46 \%$ de los casos en los centros 
públicos, y solo en el 17\% en los centros privados. En líneas generales la mayoría de los buscadores están ubicados en zonas de la pantalla de fácil localización (34 de 50 están en la zona superior o central y alineados a la derecha), lo cual indica que este parámetro está relativamente bien cuidado en los portales de las universidades españolas.

En cuanto a la accesibilidad web, solo 24 universidades (alrededor de un tercio) superan los requisitos del primer nivel. De este conjunto, una (Oviedo) satisface también el segundo nivel, y tres universidades catalanas (Politécnica, Internacional y Oberta) presentan un pequeño fallo que les impide alcanzar esa cota.

\section{Resultados concernientes a la fidelización}

Los datos de alexa.com proporcionan información sobre el tráfico dirigido a los portales, y se detecta una muy desigual distribución. Como es lógico, los portales de universidades grandes (Barcelona, Complutense, Autónoma de Barcelona) ocupan los primeros lugares, aunque también destacan por su tráfico universidades politécnicas (Madrid y Valencia) y universidades a distancia (UNED y Oberta de Catalunya).

Alrededor de la mitad de las universidades ofrecen servicios de campus virtual y de pasarela de correo electrónico desde sus páginas de inicio. Este porcentaje seguramente aumentará con el paso del tiempo. Un poco más de la mitad de los portales web definen perfiles por tipo de usuario. Solo en 3 casos se ofrece la posibilidad de personalizar el portal.

En cuanto a los servicios de acceso a la información llama la atención la complicación para encontrar los directorios de las instituciones. El uso de información multimedia es incipiente. Solo 12 universidades (alrededor del 15\%) ofrecían algún tipo de servicio de difusión de la información, normalmente vía lista de correo electrónico. El uso del RSS es muy poco frecuente debido a lo reciente de esta tecnología en la fecha de realización del estudio.

\section{Estadísticos}

A continuación se presenta un resumen de los principales estadísticos de los valores obtenidos de las cuatro medidas analizadas y del total de resultados.

En el resumen de estadísticos se observa que la medida con valores medio y máximo más altos y de mayor uniformidad es la buscabilidad. Al resto de medidas les queda aún mucho recorrido. Se ve claramente que la organización y la fidelización son los aspectos menos uniformes en su implantación en los portales y que la medida final ubicuidad y usabilidad alcanza un escaso valor medio por debajo del $50 \%$ del rango de valores $(39,73 \%$ exactamente). Su distribución es uniforme y su valor máximo alcanza el 64,76\% (Universidad Complutense de Madrid).

Scire. 14 : 2 (jul.-dic. 2008) 87-106. ISSN 1135-3716. 


\begin{tabular}{|l|c|c|c|c|c|}
\hline & Media & Máximo & Mínimo & Desviación & C. V. Pearson \\
\hline Buscabilidad & 4,25 & $6,78(7)$ & 1,83 & 1,12 & 0,26 \\
\hline Visibilidad & 2,27 & $4,66(7)$ & 0,50 & 0,98 & 0,43 \\
\hline Organización & 2,23 & $5,00(6)$ & 0,00 & 1,35 & 0,60 \\
\hline Fidelización & 1,57 & 3,77 & $-0,91$ & 1,00 & 0,63 \\
\hline Fórmula U $^{2}$ & 10,33 & $16,84(26)$ & 4,54 & 3,17 & 0,30 \\
\hline
\end{tabular}

Tabla V. Resumen de estadísticos del estudio.

Aplicar el coeficiente de asimetría de Fisher a los valores medios (totales) de la fórmula final proporciona un valor de 0,08 (muy ligera distribución asimétrica positiva). El coeficiente de Curtosis obtenido es de -0,51 (distribución platicúrtica con reducido grado de concentración alrededor de los valores centrales de la variable). En la siguiente tabla se comparan los estadísticos anteriores distinguiendo entre sector público y privado.

\begin{tabular}{|c|c|c|c|c|c|}
\hline & Media & Máximo & Mínimo & Desviación & C. V. Pearson \\
\hline Buscabilidad públicas & 4,42 & 6,78 & 1,83 & 1,10 & 0,25 \\
\hline Buscabilidad privadas & 3,89 & 6,64 & 2,11 & 1,10 & 0,28 \\
\hline Visibilidad públicas & 2,55 & 4,66 & 0,82 & 0,90 & 0,35 \\
\hline Visibilidad privadas & 1,65 & 3,24 & 0,50 & 0,84 & 0,50 \\
\hline Organización públicas & 2,42 & 5,00 & 0,00 & 1,34 & 0,55 \\
\hline Organización privadas & 1,82 & 4,50 & 0,00 & 1,23 & 0,67 \\
\hline Fidelización públicas & 1,94 & 3,77 & $-0,50$ & 0,84 & 0,43 \\
\hline Fidelización privadas & 0,77 & 3,09 & $-0,91$ & 0,82 & 1,07 \\
\hline $\mathrm{U}^{2}$ públicas & 11,34 & 16,84 & 6,01 & 2,50 & 0,22 \\
\hline $\mathrm{U}^{2}$ privadas & 8,14 & 13,87 & 4,54 & 2,57 & 0,31 \\
\hline
\end{tabular}

Tabla VI. Contraste de estadísticos entre sectores público y privado.

Las universidades públicas poseen en todos los aspectos evaluados mejores valores medios y siempre una de ellas alcanza el valor máximo. 


\section{Clasificación final}

En la tabla VII puede consultarse la clasificación final de la medida $\mathrm{U}^{2}$.

\begin{tabular}{|c|l|r|l|r|l|r|}
\hline \multicolumn{1}{|c|}{ Universidad } & \multicolumn{2}{|c|}{ Universidad } & \multicolumn{1}{c|}{ Universidad } & \\
\hline $\mathbf{1}$ & Complutense & $\mathbf{1 6 , 8 4}$ & Huelva & 11,96 & Vigo & 9,17 \\
\hline $\mathbf{2}$ & Autónoma de Barcelona & $\mathbf{1 6 , 3 8}$ & Carlos III & 11,91 & Cardenal Herrera & 9,11 \\
\hline $\mathbf{3}$ & Alicante & $\mathbf{1 6 , 0 6}$ & Pontificia Comillas & 11,68 & Pablo Olavide & 8,76 \\
\hline $\mathbf{4}$ & Granada & $\mathbf{1 5 , 1 9}$ & Illes Balears & 11,44 & País Vasco & 8,50 \\
\hline $\mathbf{5}$ & Santiago & $\mathbf{1 5 , 1 4}$ & Sevilla & 11,28 & Rey Juan Carlos & 8,48 \\
\hline $\mathbf{6}$ & Murcia & $\mathbf{1 4 , 7 6}$ & Las Palmas & 11,25 & Católica de Valencia & 8,43 \\
\hline $\mathbf{7}$ & Oviedo & $\mathbf{1 4 , 4 6}$ & Valladolid & 11,09 & Lleida & 8,37 \\
\hline $\mathbf{8}$ & Málaga & $\mathbf{1 4 , 1 3}$ & Europea de Madrid & 11,09 & Jaén & 8,32 \\
\hline $\mathbf{9}$ & Valencia & $\mathbf{1 4 , 0 3}$ & Oberta de Catalunya & 10,63 & Almería & 8,07 \\
\hline $\mathbf{1 0}$ & Navarra & $\mathbf{1 3 , 8 7}$ & Politécnica de Valencia & 10,61 & SEK & 7,71 \\
\hline Extremadura & 13,65 & Salamanca & 10,47 & La Laguna & 7,60 \\
\hline Politécnica de Cataluña & 13,06 & Politécnica de Cartagena & 10,21 & Antonio Nebrija & 7,47 \\
\hline Nacional a Distancia & 12,81 & Pública de Navarra & 10,01 & Internacional & 7,45 \\
\hline Politécnica de Madrid & 12,72 & Autónoma de Madrid & 9,85 & Camilo José Cela & 7,26 \\
\hline Deusto & 12,57 & Rovira i Virgili & 9,81 & Abat Oliba & 7,04 \\
\hline Alcalá & 12,51 & Cantabria & 9,71 & Pontificia de Salamanca & 7,04 \\
\hline Pompeu Fabra & 12,50 & Barcelona & 9,68 & San Pablo & 6,84 \\
\hline CLM & 12,37 & Mondragón & 9,68 & Alfonso X & 6,75 \\
\hline Zaragoza & 12,35 & Miguel Hernández & 9,56 & Católica de Ávila & Ávila \\
\hline Cádiz & 12,33 & Ramón Llul & 9,51 & Internacional de Andalucía & 6,01 \\
\hline La Rioja & 12,17 & A Coruña & 9,39 & San Jorge & 5,73 \\
\hline Jaume I & 12,07 & Burgos & 9,34 & Francisco de Vitoria & 5,54 \\
\hline Córdoba & 12,04 & Girona & 9,34 & Católica de Murcia & 4,73 \\
\hline León & 11,99 & Vic & 9,20 & Europea & 4,69 \\
\hline & & & & Internacional de Cataluña & 4,54 \\
\hline
\end{tabular}

Tabla VII. Clasificación final de la medida $U^{2}$ de los portales de las Universidades Españolas.

Las tablas VIII, IX, X y XI recogen las diez universidades con mejores valores por categoría estudiada. 


\begin{tabular}{|l|c|}
\hline Universidad & Puntación \\
\hline Granada & 6,78 \\
\hline Alicante & 6,68 \\
\hline Navarra & 6,64 \\
\hline León & 6,26 \\
\hline Zaragoza & 5,90 \\
\hline CLM & 5,87 \\
\hline Complutense & 5,82 \\
\hline Málaga & 5,79 \\
\hline Carlos III & 5,74 \\
\hline Huelva & 5,43 \\
\hline
\end{tabular}

\begin{tabular}{|l|c|}
\hline Universidad & Puntación \\
\hline Politécnica de Cataluña & 4,66 \\
\hline País Vasco & 4,59 \\
\hline Nacional a Distancia & 4,37 \\
\hline Autónoma de Barcelona & 4,33 \\
\hline Valencia & 3,89 \\
\hline La Rioja & 3,81 \\
\hline Barcelona & 3,72 \\
\hline Jaume I & 3,56 \\
\hline Cantabria & 3,55 \\
\hline Santiago & 3,51 \\
\hline
\end{tabular}

Tabla VIII. Top 10 de buscabilidad.

Tabla IX. Top 10 de visibilidad.

\begin{tabular}{|l|c|}
\hline Universidad & Puntación \\
\hline Autónoma de Barcelona & 5,00 \\
\hline Murcia & 4,50 \\
\hline Oviedo & 4,50 \\
\hline Santiago & 4,50 \\
\hline Deusto & 4,50 \\
\hline Alcalá & 4,00 \\
\hline Alicante & 4,00 \\
\hline Cádiz & 4,00 \\
\hline Complutense & 4,00 \\
\hline Extremadura & 4,00 \\
\hline
\end{tabular}

\begin{tabular}{|l|c|}
\hline Universidad & Puntación \\
\hline Complutense & 3,77 \\
\hline Málaga & 3,61 \\
\hline Oviedo & 3,53 \\
\hline Las Palmas & 3,29 \\
\hline Nacional a Distancia & 3,17 \\
\hline Oberta de Catalunya & 3,09 \\
\hline Politécnica de Valencia & 2,82 \\
\hline Huelva & 2,75 \\
\hline Politécnica de Madrid & 2,72 \\
\hline Granada & 2,66 \\
\hline
\end{tabular}

Tabla X. Top 10 de organización.

Tabla XI. Top 10 de fidelización.

La tabla XII muestra las primeras universidades en cada de las categorías estudiadas y el porcentaje sobre el total de puntos posibles en la misma que ha obtenido cada una de ellas.

\begin{tabular}{|l|c|c|c|}
\hline Categoría & Universidad & Puntación & $\%$ \\
\hline Buscabilidad & Granada & 6,78 & 0,96 \\
\hline Visibilidad & Politécnica de Cataluña & 4,66 & 0,66 \\
\hline Organización & Autónoma de Barcelona & 5,00 & 0,83 \\
\hline Fidelización & Complutense & 3,77 & 0,62 \\
\hline
\end{tabular}

Tabla XII. Top resumen.

Scire. $14: 2$ (jul.-dic. 2008) 87-106. ISSN 1135-3716. 
Se observa que ninguna universidad ha conseguido ocupar el primer lugar en dos categorías de parámetros al mismo tiempo.

\section{Conclusiones}

Este estudio demuestra que es viable evaluar la calidad de diseño de un portal web por medio de la selección e identificación de una serie de indicadores cuantitativos distantes de valoraciones subjetivas. Es un punto de partida para posteriores análisis y revisiones de los parámetros estudiados y de los indicadores elegidos. No se trata de un trabajo aislado, ya que nace con una voluntad abierta de fomentar el diálogo y la discusión en torno a este en el seno de una comunidad científica y técnica que en los últimos años ha venido demostrando un gran interés en el mismo.

Los trabajos llevados a cabo hasta ahora sobre la totalidad de los portales solo analizaban un aspecto determinado. Igualmente, aquellos estudios desarrollados en profundidad únicamente se habían ocupado de una porción del conjunto total de portales. Se ha detectado que los investigadores conceden gran importancia a aspectos específicos de la ubicuidad y de la usabilidad, y resulta gratificante el interés suscitado hacia esta temática en el área de biblioteconomía y documentación.

Se han detectado algunas conceptualizaciones ligeramente diferentes de la visibilidad. A los portales universitarios aún les queda bastante recorrido para alcanzar el grado máximo de calidad, con excepción quizá de los aspectos vinculados con la buscabilidad. El nivel medio de desarrollo de los portales universitarios españoles del sector público es superior al de los del sector privado. Ningún portal ocupa el primer lugar de forma simultánea en dos o más categorías de parámetros. Esto tiene que ver con la complementariedad y exhaustividad de los indicadores analizados.

A pesar de algunas medidas correctoras, el estado actual de la accesibilidad de los portales universitarios es deplorable. Aún no se han abolido ciertos hábitos desaconsejables en el diseño web. El uso de los metadatos es bastante escaso y muy desigual.

A modo de colofón nos atrevemos a comentar que nuestra medida global de la calidad de los portales $\left(\mathrm{U}^{2}\right)$ ha ofrecido unos resultados consistentes y coincidentes con los obtenidos en algunos aspectos parciales de otros estudios, por lo que creemos haber comenzado una línea de trabajo simplificadora e integradora que nos permitirá continuar midiendo la evolución de estos portales. Igualmente, no resulta del todo desdeñable pensar en aplicar esta metodología de análisis a otro tipo de comunidades de portales de la Web, con lo cual aportaremos, como fruto de una investigación aplicada y focalizada en el mundo universitario, una nueva herramienta a toda la comunidad. 


\section{Referencias}

Abadal Falgueras, E.; Rius Alcaraz, L. (2006). Revistas científicas digitales: características e indicadores. // Revista de Universidad y Sociedad del Conocimiento. 3:1 (abril 2006). http://www.uoc.edu/rusc/3/1/dt/esp/abadal_rius.pdf (2006-04-08).

Aguilló, I. F.; Granadino, B. (2006). Indicadores web para medir la presencia de las universidades en la Red. // Revista de Universidad y Sociedad del Conocimiento. 3:1 (abril 2006). http://www.uoc.edu/rusc/3/1/dt/esp/aguillo_granadino.pdf (2006-04-08).

Baeza-Yates, R. (2002). 5 claves para la web. http://www.dcc.uchile.cl/ rbaeza/inf/ reglasweb.html (2006-02-02).

Baeza-Yates, R.; Rivera Loaiza, C. (2002). Ubicuidad y usabilidad en la red. http://www.dcc.uchile.cl/ rbaeza/inf/usabilidad.html (2006-02-02).

Craven, T. C. (2004). Variations in use of meta tag descriptions by web pages in different languages. // Information Processing and Management. 40 (2004) 479-493.

disc@ @net (2004). La accesibilidad en los portales universitarios: evaluación técnica de la accesibilidad y valoración de la experiencia de usuario en 15 portales de universidades españolas. http://www.discapnet.es/inc/infoaccesibilidad/html/Portales_universitarios_ sintética.htm (2005-10-24).

disc@pnet (2006). Accesibilidad de portales web universitarios, febrero de 2006. http://www.discapnet.es/Discapnet/Castellano/Observatorio_infoaccesibilidad/ Accesibilidad+de+Portales+Web+Universitarios+2006.htm (2006-04-17).

Duart, J. M. (2006). La Universidad en la Red. // Revista de Universidad y Sociedad del Conocimiento. 3:1 (abril 2006). http://www.uoc.edu/rusc/3/1/editorial.html (2006-04-08).

Dublin Core Metadata Initiative (2005). DCMI Metadata Terms. http://dublincore.org/ documents/demi-terms/\#H2 (2005-10-30).

Franklin, T. (2006). Portales en la educación superior: conceptos y modelos. // Revista de Universidad y Sociedad del Conocimiento. 3:1 (abril 2006). http://www.uoc.edu/rusc/ 3/1/dt/esp/franklin.pdf (2006-04-07).

García Gómez, J. C. (2005). La accesibilidad de los portales universitarios. // Úsalo: usabilidad para todos. http://usalo.es/45/la-accesibilidad-de-los-portales-universitarios/ (2006-04-04).

García Gómez, J. C. (2006). Motivos para hacer páginas accesibles. // Úsalo: usabilidad para todos. http://usalo.es/130/motivos-para-hacer-paginas-accesibles/ (2006-04-04).

Gil Leiva, I.; Moya Martínez, G. (2001). Evaluación de websites de Universidades Españolas. // Actas InterMedia 2001: Internacional Workshop on Multimedia Applications (CD-ROM).

LabInternet (2006). Ranking cibermétrico de las universidades españolas.: http://www. webometrics.info/top100_spain_es.asp.htm (2006-03-06).

Morville, P. (2005). Ambient findability. Cambridge, MA: MIT Press, 2005.

Nielsen, J.; Tahir, M. (2002). Usabilidad de páginas de inicio: análisis de 50 sitios web. Madrid [etc.]: Pearson, 2002.

Nielsen, Jakob (2003). Usability 101: Introduction to Usability. // useit.com. (25 Aug 2003). http://www.useit.com/alertbox/20030825.html

Scire. 14 : 2 (jul.-dic. 2008) 87-106. ISSN 1135-3716. 
Pinto Molina, M., et álii (2004). Análisis cualitativo de la visibilidad de la investigación de las universidades españolas a través de sus páginas web. // Revista Española de Documentación Científica. 27:3 (2004) 345-373.

Rosenfeld, L.; Morville, P. (2000). Arquitectura de información para el WWW. México [etc.]: Mc.Graw-Hill, 2000.

AIPO (2005). USABAIPO: Proyecto de Usabilidad en Universidades Españolas. http://griho.udl.es/usabilidad/4NovaUsabPagWeb/paginas/index.jsp (2005-10-22).

World Wide Web Consortium (1999). Web Content Accessibility Guidelines 1.0. http://www.w3.org/TR/WAI-WEBCONTENT/ (2005-11-11).

World Wide Web Consortium (2005). Web Content Accessibility Guidelines 2.0. http://www.w3.org/TR/2005/WD-WCAG20-20051123/ (2005-11-30).

Zhang, J.; Dimitroff, A. (2005). The impact of metadata implementation on webpage visibility in search engine results (part II). // Information Processing and Management. 41 (2005) 691-715.

Zhang, J.; Jastram, I. (2006). A study of the metadata creation behaviour of different user groups on the Internet. // Information Processing and Management. 42 (2006) 10991122. 